Pacific Journal of Mathematic 


\title{
ON CERTAIN SERIES EXPANSIONS INVOLVING WHITTAKER FUNCTIONS AND JACOBI POLYNOMIALS
}

\author{
Peter Henrici
}

\section{INTRODUCTION}

1. 1. Outline of the paper. By substituting polar coordinates in the partial differential equation

$$
\frac{\partial^{2} u}{\partial x^{2}}+\frac{\partial^{2} u}{\partial y^{2}}+\begin{array}{cc}
4 \mu+1 & \partial u \\
x & \partial x
\end{array}+\begin{gathered}
4 \nu+1 \\
y
\end{gathered} \frac{\partial u}{\partial y}+k\left[4 \lambda-k\left(x^{2}+y^{2}\right)\right] u=0
$$

and separating variables, one is led in a natural way to certain combinations of Whittaker functions and Jacobi polynomials (called for brevity J.-W. functions in this paper). With a view towards deriving some functional relations involving hypergeometric functions, we develop in the first part of the paper a technique for the construction of expansions of arbitrary regular analytic solutions of (1) in terms of these J.-W. functions. The method of our investigation consists in setting up a one-to-one correspondence between the class of even analytic functions of one complex variable regular in a circle around the origin and a certain class $E$ of regular solutions of (1). This correspondence associates with a solution $u(x, y) \in E$ the function $u(x,-i x)$ obtained by considering $u(x, y)$ on the (imaginary) characteristic $x-i y=0$ of (1). ${ }^{1}$ Since the maps of the even powers of a single variable in this correspondence are shown to be the J.-W. functions mentioned above, the expansion problem in question is reduced to the problem of finding the Taylor expansion of a given analytic function of one variable.

Applying this technique to some special solutions of (1), we are led to three expansions involving various kinds of hypergeometric functions. The first of them contains a number of well-known theorems on special functions as special cases, namely, among others, Bateman's addition theorem in the theory of Bessel functions, Ramanujan's formula for the product of two confluent hypergeometric series, and Erdélyi's addition theorem (with respect to the parameters) for the product of two $M$-functions. The second application gives rise to

Received October 9, 1953. This paper was prepared under a National Bureau of Standards contract with American University. The author is indebted to Prof. A. Erdélyi, who saw a first draft of this paper, for some most helpful critical remarks.

1 This procedure is related to Bergman's operator method in the theory of elliptic partial differential equations with regular coefficients; see the remark at the end of $\$ 4$. 
another addition formula (in the ordinary sense) for the product of two $M$-functions, while the third may be looked at as an alternate formulation of Bailey's decomposition formula for a special case of Appell's function $F_{4}$.

1. 2. Definitions. In (1) the parameters $\mu, \nu, \lambda, k$ are arbitrary complex numbers with the only exception that $\mu$ and $\nu$ are subject to the condition

$$
2 \mu+2 \nu \neq-2,-3,-4, \cdots .
$$

Sets of values $(\mu, \nu, \lambda, k)$ satisfying (2) are called admissible values of the parameters.

If $\mathscr{B}$ denotes a domain of the complex $(x, y)$-space which contains the origin, we denote by $E_{\mathscr{B}}$ the class of analytic functions $u(x, y)$ of the two complex variables $x$ and $y$ which

(i) are regular in $\mathscr{B}$,

(ii) are even fuctions of $x$ and of $y$, and

(iii) satisfy (1) for certain admissible values of the parameters. ${ }^{2}$

We denote by $\mathscr{K}_{r}$, the circle $|z|<r$ of the complex $z$-plane, and by $\mathscr{K}_{r} \times \mathscr{K}_{r}$ the bicylinder $|z|<r,\left|z^{*}\right|<r$ in the space $K^{2}$ of the two complex variables $z$ and $z^{*}$.

Our notation of special functions follows the traditional lines. For the ordinary and the generalised hypergeometric series we found it convenient to use Bailey's notation [1, p. 8]

\section{JACOBI-WhitTAKer FunCTIONS}

Our first aim is to construct a set of solutions of (1) by the elementary method of separating variables. Introducing in (1) the new variables

$$
\rho=x^{2}+z^{2}, \quad \tau=\frac{x^{2}-y^{2}}{x^{2}+y^{2}},
$$

we obtain for $v(\rho, \tau)=u(x, y)$ the equation

$$
\begin{aligned}
\rho^{2} \frac{\partial^{2} v}{\partial \rho^{2}}+2(1+\mu+\nu) \rho & \frac{\partial v}{\partial \rho} \\
+ & \left(1-\tau^{2}\right) \frac{\partial^{2} v}{\partial \tau^{2}} \\
& +2[\mu-\nu-(1+\mu+\nu) \tau] \frac{\partial v}{\partial \tau}+k \rho\left(\lambda-\frac{k \rho}{4}\right) v=0 .
\end{aligned}
$$

If $v(\rho, \tau)=R(\rho) T(\tau)$ is a solution of (4), one finds by the usual sepa-

${ }^{2} \mathrm{We}$ do not investigate the problem of the extent to which the three conditions (i), (ii), and (iii) imply each other. 
ration method that $R(\rho)$ and $T(\tau)$ have to satisfy separately the equations

$$
\frac{d^{2} R}{d \rho^{2}}+2(1+\mu+\nu) \frac{1}{\rho} \frac{d R}{d \rho}+\left[-\frac{s}{\rho^{2}}+\frac{k \lambda}{\rho}-\frac{k^{2}}{4}\right] R=0,
$$

and

$$
\left(1-\tau^{2}\right) \frac{d^{2} T}{d \tau^{2}}+2[\mu-\nu-(1+\mu+\nu) \tau] \frac{d T}{d \tau}+s T=0,
$$

where $s$ is a separation parameter. Writing $s=n(2 \mu+2 \nu+1+n)$, we find that solutions of (5) which are regular near $\rho=0$ are represented for $n=0,1,2, \cdots$ by

$$
\begin{aligned}
R(\rho) & =\rho^{-\mu-\nu-1} M_{\lambda, \mu+\nu+\frac{1}{2}+n}(k \rho) \\
& =k^{\mu+\nu+1+n} \rho^{n} e^{-\frac{k \rho}{2}} F_{1} F_{1}\left[\begin{array}{l}
\mu+\nu+1+n-\lambda ; k \rho \\
2 \mu+2 \nu+2+2 n
\end{array}\right],
\end{aligned}
$$

where $M$ denotes the Whittaker function of the first kind, while (6) has for the same values of $s$ the polynomial solution

$$
\begin{aligned}
& T(\tau)=P_{n}^{(2 \nu, 2 \mu)}(\tau) \\
& =\frac{(2 \nu+1)_{n}{ }_{2} F_{1}}{n !}\left[\begin{array}{l}
-n, 2 \mu+2 \nu+1+n ; \\
2 \nu+1
\end{array}, \begin{array}{c}
1-\tau \\
2
\end{array}\right],
\end{aligned}
$$

where $P$ stands for the Jacobi polynomial in the notation of Szegö [11, p. 61]. Provided (2) is valid, solutions of (1) regular near $x=y=0$ are thus given by the functions

$$
f_{n}^{(\mu, \nu)}(\rho, \tau ; \lambda, k)=c_{n} P_{n}^{(i \nu, 2 \mu)}(\tau) k^{-n}(k \rho)^{-\mu-\nu-1} M_{\lambda, \mu+\nu+\frac{1}{2}+n}(k \rho)
$$

where

$$
c_{n}=\frac{2^{2 n} n !}{(2 \mu+2 \nu+1+n)_{n}}=\frac{(2 \mu+2 \nu+1)_{n} n !}{\left(\mu+\nu+\frac{1}{2}\right)_{n}(\mu+\nu+1)_{n}}
$$

is a normalisation factor introduced for later convenience. We shall call these functions for brevity Jacobi-Whittaker functions (J.-W. functions) of order $n$. The arguments $\lambda$ and $k$ in $f_{n}^{(\mu, \nu)}$ will usually be omitted, if it is not necessary to exhibit them explicitly.

For later reference we note the following special and limiting cases of the functions $f_{n}^{(\mu, \nu)}$ : 
(i) For $\lambda=0$ we have $[6, p .13]$

$$
\begin{aligned}
& f_{n}^{(\mu, \nu)}(\rho, \tau ; 0 ; k) \\
& \quad=c_{n} P_{n}^{(2 \nu, 2 \mu)}(\tau) I^{\prime}\left(\mu+\nu+\frac{3}{2}+n\right)\left(\frac{k}{4}\right)^{-n}\left(\frac{k \rho}{4}\right)^{-\mu-\nu-\frac{1}{2}} I_{\mu+\nu+\frac{1}{2}+n}\left(\frac{k \rho}{2}\right),
\end{aligned}
$$

where $I$ is a modified Bessel function.

(ii) Putting $\lambda=\frac{\kappa}{4 k}$ and letting $k \rightarrow 0$, we obtain the function

$$
\begin{aligned}
& f_{n}^{(\mu, \nu)}(\rho, \tau ; \kappa)=\lim _{\mathrm{k} \rightarrow 0} f_{n}^{(\mu, \nu)}\left(\rho, \tau, \frac{\kappa}{4 k}, k\right) \\
& \quad=c_{n} P_{n}^{(2 \nu, 2 \mu)}(\tau) \Gamma(2 \mu+2 \nu+2+2 n)\left(\frac{\kappa}{4}\right)^{-n}\left(\frac{\kappa \rho}{4}\right)^{-\mu-\nu-\frac{1}{2}} J_{2 \mu+2 \nu+1+2 n}(\sqrt{\kappa \rho})
\end{aligned}
$$

where $J$ denotes the ordinary Bessel function. Evidently (12) satisfies the differential equation

$$
\frac{\partial^{2} u}{\partial x^{2}}+\frac{\partial^{2} u}{\partial y^{2}}+\frac{4 \mu+1}{x} \frac{\partial u}{\partial x}+\frac{4 \nu+1}{y} \frac{\partial u}{\partial y}+\kappa u=0 .
$$

We will refer to (12) as to the "reduced" case of the functions $f_{n}^{(\mu, \nu)}$. The limiting values of $\lambda$ and $k$ leading to it are included in the admissible values of the parameters.

(iii) For $\lambda=k=0$ we have from (9), (11) or (12)

$$
f_{n}^{(\mu, \nu)}(\rho, \tau ; 0,0)=c_{n} \rho^{n} P_{n}^{(2 \nu, 2 \mu)}(\tau) .
$$

We study next some properties of the J.-W. functions considered as functions of the two complex variables $z$ and $z^{k}$ defined by

$$
z=x+i y, \quad z^{*}=x-i y .^{3}
$$

As such they satisfy the differential equation

(16) $\frac{\partial^{2} U}{\partial z \partial z^{*}}+\frac{2 \mu+\frac{1}{2}}{z+z^{*}}\left\{\frac{\partial U}{\partial z}+\frac{\partial U}{\partial z^{*}}\right\}-\frac{2 \nu+\frac{1}{2}}{z-z^{*}}\left\{\frac{\partial U}{\partial z}-\frac{\partial U}{z^{*}}\right\}+k\left[\lambda-k \frac{z z^{*}}{4}\right] U=0$,

which is readily constructed by inserting in (1) the variables (15). From (3) it is evident that

$$
\rho=z z^{*}, \quad \tau=\frac{z^{2}+z^{* 2}}{2 z z^{*}} .
$$

Thus we have for

$$
F_{n}^{(\mu, \nu)}\left(z, z^{*}\right)=f_{n}^{(\mu, \nu)}(\rho, \tau)
$$

${ }^{3}$ It is assumed throughout the paper that $x$ and $y$ are independent complex variables, so that also $z$ and $z^{*}$ take on independent complex values. 
the representation

$$
\begin{aligned}
& F_{n}^{(\mu, \nu)}\left(z, z^{*}\right) \\
& \quad=c_{n} P_{n}^{(2 \nu, 2 \mu)}\left(\begin{array}{c}
z^{2}+z^{* 2} \\
2 z z^{*}
\end{array}\right) k^{-n}\left(k z z^{*}\right)^{-\mu-\nu-1} M_{\lambda, \mu+\nu+\frac{1}{2}+n}\left(k z z^{*}\right) .
\end{aligned}
$$

Using the relations $[11, \mathrm{pp} .58,61]$

$$
P_{n}^{(2 \nu, 2 \mu)}(\tau)=(-)^{n} P_{n}^{(2 \mu, 2 \nu)}(-\tau)
$$

and

$$
P_{n}^{\left({ }^{(2 \mu, 2 \nu)}(\tau)\right.}=\frac{(2 \mu+2 \nu+1+n)_{n}}{n !}\left(\frac{\tau-1}{2}\right)^{n}{ }_{2} F_{1}\left[\begin{array}{c}
-n,-n-2 \mu ; \\
-2 n-2 \mu-2 \nu \\
-2-\tau
\end{array}\right]
$$

and observing (7), we may write this also as follows:

$$
\begin{aligned}
& F_{n}{ }^{(\mu, \nu)}\left(z, z^{*}\right) \\
& \quad=\left(z+z^{*}\right)^{2 n}{ }_{2} F_{1}\left[\begin{array}{cc}
-n,-n-2 \mu ; & 4 z z^{*} \\
-2 n-2 \mu-2 \nu & \left(z+z^{*}\right)^{2}
\end{array}\right] e^{-\frac{k z z^{*}}{2}{ }_{1} F_{1}\left[\begin{array}{ll}
\mu+\nu+1+n-\lambda ; & k z z^{*} \\
2 \mu+2 \nu+2+2 n
\end{array}\right] .}
\end{aligned}
$$

From this representation it is easy to draw the following conclusions :

Lemma 1. For all admissible values of the parameters,

$$
F_{n}{ }^{(\mu, \nu)}\left(z, z^{*}\right) \in E_{K^{2}} .
$$

LEMMA 2. For all admissible values of the parameters,

$$
F_{n}^{(\mu, \nu)}(z, 0)=z^{2 n} \text {. }
$$

In order to prove Lemma 1 we observe that the last two factors in (18) are entire functions of $z z^{*}$, while, since the series ${ }_{2} F_{1}$ in (18) terminates after at most $n$ terms, the first two factors form together a polynomial in $z$ and $z^{*}$. The solution (18) of (16) is thus an entire function of $z$ and $z^{*}$. Furthermore the conditions of symmetry imposed on the elements of $E$, which for functions of $z$ and $z^{*}$ amount to the relations

$$
F\left(z, z^{*}\right)=F\left(-z,-z^{*}\right), \quad F\left(z, z^{*}\right)=F\left(z^{*}, z\right),
$$

are satisfied by (18). Lemma 2 follows simply from the fact that for $z^{*}=0$ the last three factors in (18) reduce to 1 . It is easy to see that both Lemma 1 and Lemma 2 remain also true in the reduced case.

We come now to a simple equiconvergence property of series of J.-W. functions.

Lemma 3. Let $r>0$ and let 


$$
f(z)=\sum_{n=0}^{\infty} a_{n} z^{2 n}
$$

be regular in $\mathscr{K}_{r}$. Then for fixed admissible values of the parameters the series

$$
F\left(z, z^{*}\right)=\sum_{n=0}^{\infty} a_{n} F_{n}^{(\mu, \nu)}\left(z, z^{*} ; \lambda, k\right)
$$

converges uniformly in every closed subregion $\mathscr{D}$ of $\mathscr{B}=\mathscr{K}_{r} \times \mathscr{K}_{r}$ and represents there a function $\in E_{\mathscr{B}}$ with the property

$$
F(z, 0)=f(z) \text {. }
$$

Proof. Obviously the second statement of the lemma follows immediatly from the first and from Lemma 2. In order to prove the uniform convergence, we again use for the J.-W. functions the representation (18). It follows in the general case from a well-known theorem on $M$-functions [8, p. 93] and in the reduced case from an analogous theorem on Bessel functions [13, p. 44, formula (1)] that for bounded $\left(z, z^{*}\right)$ and for $n$ large the product of the last two factors in (18) is asymptotically equal to 1 . It suffices therefore to consider the case $\lambda=k=0$. We make now use of the well-known generating function of the Jacobi polynomials [11, p. 68. formula (4.4.5)]. Replacing the variables $\alpha, \beta, x, w$ in Szegö's formula by $2 \nu, 2 \mu,\left(z^{2}+z^{* 2}\right) / 2 z z^{*}, t z z^{*}$ respectively and observing (17), we obtain the power series in $t$

$$
\sum_{n=0}^{\infty} c_{n}{ }^{-1} F_{n}{ }^{(\mu, \nu)}\left(z, z^{*} ; 0,0\right) t^{n}=E\left(z, z^{*} ; t\right),
$$

where for given $r^{\prime \prime}>0, E\left(z, z^{*} ; t\right)$ is a certain analytic function of $z$, $z^{*}$ and $t$ regular in $\left(z, z^{*}\right) \in\left\{\mathscr{K}_{r^{\prime \prime}} \times \mathscr{K}_{r^{\prime \prime}}\right\} \cap\left\{|t|<r^{\prime \prime-2}\right\}$. Let now $\mathscr{D}$ be enclosed in a bicylinder $\mathscr{K}_{r^{\prime}} \times \mathscr{K}_{r^{\prime}}$, where $r^{\prime}<r$, and choose $r^{\prime \prime}, r^{\prime \prime \prime}$ such that $r^{\prime}<r^{\prime \prime}<r^{\prime \prime \prime}<r$. Applying to (23) Cauchy's estimate for the coefficients of a power series with $|t|=r^{\prime \prime \prime-2}$ yields

$$
\left|F_{n}^{(\mu, \nu)}\left(z, z^{*} ; 0,0\right)\right| \leq K\left|c_{n}\right| r^{\prime \prime 2,2},
$$

where

$$
K=\max _{\substack{|t|=r^{\prime \prime \prime}-2 \\\left(z, z^{*}\right) \in \mathcal{K}_{r^{\prime}} \times \mathcal{K}_{r^{\prime}}}}\left|E\left(z, z^{*} ; t\right)\right|
$$

is finite and does not depend on $n$. Therefore the terms of (21) are dominated in $\mathscr{D}$ by the terms of the series

$$
K \sum_{n=0}^{\infty}\left|a_{n}\right|\left|c_{n}\right| r^{\prime 22 n},
$$


which converges absolutely, since $\left|c_{n}\right| c_{n-1} \mid \rightarrow 1(n \rightarrow \infty)$ and (20) converges for some $z=r^{\prime \prime \prime \prime}$ with $r^{\prime \prime \prime}<r^{\prime \prime \prime \prime}<r{ }^{4}$

\section{A Uniqueness Theorem for Solutions of (1)}

Lemma 4. Let $\varnothing$ be a domain $\subset K^{2}$, containing the origin, and let $F\left(z, z^{*}\right)$ be a function $\in E$ such that $F(z, 0)=0$. Then $F\left(z, z^{*}\right)=0$ in $\mathscr{D}$.

Remark. The proof of this lemma does not follow from the general uniqueness theorems for hyperbolic initial value problems (see, for example, [7, p. 321]), since some of the coefficients in (16) are singular.

Procf. In view of the relations (19) the power series expansion of $F$, which by assumption converges in a certain neighbourhood of the origin, must be of the form

$$
F\left(z, z^{*}\right)=\sum_{m=0}^{\infty} \sum_{n=0}^{m} c_{m, n} z^{2 m-n} z^{* n},
$$

where

$$
c_{m, n}=c_{m, 2 m-n} .
$$

If we call $s+t$ the weight of the monomial $z^{s} z^{* t}$, we may say that (25) contains only terms of even weight. By assumption and by (26),

$$
c_{m, 0}=c_{m, 2 m}=0 \text {, }
$$$$
m=0,1,2, \cdots \text {. }
$$

By differentiating (25) and substituting into (16) we obtain, after multiplying by $z^{2}-z^{* 2}$,

$$
\begin{gathered}
\sum_{m=0}^{\infty} \sum_{n=0}^{m} c_{m, n}\left\{n(2 m-n-2 \mu-2 \nu-1) z^{2 m-n+1} z^{* n-1}+4(m-n)(\mu-\nu) z^{2 m-n} z^{* n}\right. \\
\left.-(m-n)(n+2 \mu+2 \nu+1) z^{2 m-n-1} z^{* n+1}+R_{m}\right\}=0
\end{gathered}
$$

where the symbol $R_{m}$ denotes terms of higher weight than $m$. We prove now that $c_{m, n}=0$ for all values of $m$ and $n$ in question by induction with respect to the weight.

By (27), $c_{0,0}=0$. Let us assume that we have proved

$$
c_{k, n}=0 \text { for } n=0,1, \cdots, 2 k ; k=0,1, \cdots, m-1 \text {. }
$$

Consider now in (28) the terms of fixed weight $m$. Then the terms $R_{m}$ will be multiplied by coefficients $\mathrm{c}_{k, n}$ with $k<m$, which are zero by

${ }^{4}$ The author is indebted to a referee for the following remark: Using the theorems about the growth of a power series of one complex variable whose coefficients satisfy certain conditions, one could obtain bounds for the functions (21) in terms of the coefficients $a_{n}$. 
(29). Considering now the fact that the coefficients of each fixed power $z^{2 m-n} z^{* n}$ must vanish separately, we are led to the recurrence relations

$$
\begin{gathered}
c_{m, 1}(m+\mu+\nu)+2 c_{m, 0} m(\mu-\nu)=0, \\
c_{m, n+1}(n+1)(2 m-n+2 \mu+2 \nu)+4 c_{m, n}(m-n)(\mu-\nu) \\
-c_{m, n-1}(2 m-n-1)(n+2 \mu+2 \nu)=0, \quad n=1,2, \cdots, 2 m-1 .
\end{gathered}
$$

Since $c_{m, 0}=0$ and since $m+\mu+\nu \neq 0$ for admissible values of the parameters, we have from (30) $c_{m, 1}=0$ and hence from (31) $c_{m, n+1}=0$ as long as $2 m-n+2 \mu+2 \nu \neq 0$, for $n=1,2, \cdots, 2 m-1$. It follows that (29) is true for $k=m$ and hence for all $k$.

\section{ExPANSION THEOREM}

The following theorem, which will be the principal tool for the special functions work in the later part of this paper, is now easy to prove.

TheOREM. Let $r>0, \mathscr{P}=\mathscr{Y}_{r} \times \mathscr{K}_{r}$ and let $F\left(z, z^{*}\right) \in E_{\mathscr{B}}$. If

$$
F(z, 0)=\sum_{n=0}^{\infty} a_{n} z^{2 n},
$$

then the series

$$
\sum_{n=0}^{\infty} a_{n} F_{n}^{(\mu, \nu)}\left(z, z^{*}\right)
$$

(which by Lemma 3 converges in $\mathscr{P}$ ) is equal to $F\left(z, z^{*}\right)$ in $\mathscr{P}$.

Proof. By Lemma 3, (33) represents a function $\in E_{\mathcal{B}}$ which is equal to $F(z, 0)$ for $z^{*}=0$. By Lemma 4 the function

$$
F\left(z, z^{*}\right)-\sum_{n=0}^{\infty} a_{n} F_{n}^{(\mu, \nu)}\left(z, z^{*}\right)
$$

vanishes identically in $\mathscr{P}$.

The expansion (33) will sometimes be called J.-W. expansion of $F\left(z, z^{*}\right)$. The function (32), the knowledge of which is sufficient for the construction of the J.-W. expansion of $F\left(z, z^{*}\right)$, will be called the generating function of this expansion.

Remark. For fixed admissible values of the parameters Lemma 3 sets up a mapping of the class of even analytic functions of a single complex variable regular in a $\mathscr{K}_{r}$ on the class $E_{\mathcal{K}_{r}} \times \mathcal{K}_{r}$. This mapping is one-to-one by Lemma 4 . The inverse mapping is given by the formula

$$
f(z)=F(z, 0),
$$


which is essentially identical with the inversion formula for Bergman's so-called integral operator of the first kind, ${ }^{5}$ whose existence, however, has been established in general only for the case where the coefficients of the differential equation are regular analytic functions in the considered domain. Our theory presents an example of a representation of an operator analogous to that of Bergman in a case where the considered differential equation has singular coefficients. ${ }^{6}$

We proceed now to construct explicity by our method the J.-W. expansions of several special solutions of (1), which are again obtained by the method of separation of variables.

\section{Applications of the Expansion Theorem : Cartesian CoORdinates}

If the function $u(x, y)=X(x) Y(y)$ is introduced in (1) (with $k=1$ ), we find that the differential equation is satisfied if $X$ and $Y$ satisfy separately the equations

$$
\begin{aligned}
& d^{2} X+4 \mu+1 \\
& d x^{2} \\
& x \quad d X \\
& d^{2} Y \\
& d y^{2}
\end{aligned}
$$

provided $\alpha+\beta=\lambda$.

Solutions of these equations which are regular near $x=0$ and $y=0$ can again be expressed by means of Whittaker functions. In view of the differential equation satisfied by these functions it is readily verified that, provided none of the numbers $2 \mu$ and $2 \nu$ is a negative integer, one may put

$$
\begin{aligned}
& X(x)=x^{-2 \mu-1} M_{\alpha, \mu}\left(x^{2}\right), \\
& Y(y)=y^{-2 \nu-1} M_{\beta, \nu}\left(y^{2}\right) .
\end{aligned}
$$

Introducing the variables $z$ and $z^{*}$ and passing to hypergeometric series we have

$$
\begin{aligned}
& u\left(\frac{z+z^{*}}{2}, \frac{z-z^{*}}{2 i}\right)=U\left(z, z^{*}\right) \\
& \quad=e^{-\frac{z z^{*}}{2}{ }_{1} F_{1}}\left[\begin{array}{l}
\mu+\frac{1}{2}-\alpha ; \frac{\left(z+z^{*}\right)^{2}}{4} \\
2 \mu+1
\end{array}\right]_{1} F_{1}\left[\begin{array}{c}
\nu+\frac{1}{2}-\beta ;-\frac{\left(z-z^{*}\right)^{2}}{4} \\
2 \nu+1
\end{array}\right] .
\end{aligned}
$$

${ }^{5}$ See [2, p. 117]. Contrary to the situation described there, our operator maps functions $f(z)$ which are real for real $z$ on solntious of (1) which are real for real $x$ and $y$.

'Other cases of differential equations with singular coefficients have been treated by Bergman [3, 4]. The "reduced" equation (13) has in the case $4 \mu+1=0$ been considered by the present author in [10], where a different method has been used. 
The generating function of the J.-W. expansion of $U\left(z, z^{*}\right)$ is thus given by

$$
\begin{aligned}
U(z, 0) & ={ }_{1} F_{1}\left[\begin{array}{l}
\mu+\frac{1}{2}-\alpha ;-\frac{z^{2}}{4} \\
2 \mu+1
\end{array}\right]_{1} F_{1}\left[\begin{array}{l}
\nu+\frac{1}{2}-\beta ;-z^{2} \\
2 \nu+1
\end{array}\right] \\
& =\sum_{m=0}^{\infty}\left(\begin{array}{l}
z^{2} \\
4
\end{array}\right)^{m} \sum_{n=0}^{m}\left(\mu+\frac{1}{2}-\alpha\right)_{m}(-2 \mu-m)_{n}\left(\nu+\frac{1}{2}-\beta\right)_{n}(-m)_{n} \\
& =\sum_{m=0}^{\infty}\left(-\mu+\alpha-m+\frac{1}{2}\right)_{n}(2 \mu+1)_{m}(2 \nu+1)_{n} m ! n ! \\
(2 \mu+1)_{m} m ! & (\mu)_{3} F_{2}\left[\begin{array}{l}
-2 \mu-m, \nu+\frac{1}{2}-\beta,-m ; \\
-\mu+\alpha-m+\frac{1}{2}, 2 \nu+1
\end{array}\right]\left(\begin{array}{c}
z^{2} \\
4
\end{array}\right)^{m} .
\end{aligned}
$$

Applying the expansion theorem, writing the J.-W. functions in the form (9) and using the relations (following from (3))

$$
x^{2}=\rho \begin{gathered}
1+\tau \\
2
\end{gathered}, \quad y^{2}=\rho \begin{gathered}
1-\tau \\
2
\end{gathered}
$$

we obtain the following J.-W. expansion for the product of two Whittaker functions with different pairs of indices and arguments, which is valid for unrestricted values of $\rho, \tau, \alpha, \beta$, as long as none of the numbers $2 \mu, 2 \nu$ and $2 \mu+2 \nu+1$ is a negative integer:

$$
\begin{gathered}
\left(\begin{array}{c}
1+\tau \\
2
\end{array}\right)^{-\mu-\frac{1}{2}} M_{\alpha, \mu}\left(\begin{array}{c}
1+\tau \\
2
\end{array}\right) \cdot\left(\rho \frac{1-\tau}{2}\right)^{-\nu-\frac{1}{2}} M_{\beta, \nu}\left(\rho \frac{1-\tau}{2}\right) \\
=\sum_{m=0}^{\infty} \frac{\left(\mu+\frac{1}{2}-\alpha\right)_{m}}{(2 \mu+1)_{m}(2 \mu+2 \nu+1+m)_{m}{ }_{3} F_{2}}\left[\begin{array}{c}
-2 \mu-m, \nu+\frac{1}{2}-\beta,-m ; \\
-\mu+\alpha-m+\frac{1}{2}, 2 \nu+1
\end{array}\right] \\
\times P_{n}^{(2 \nu, 2 \mu)}(\tau) \rho^{-\mu-\nu-1} M_{\alpha+\beta, \mu+\nu+\frac{1}{2}+m}(\rho) .
\end{gathered}
$$

This mother expansion has a great number of children and grandchildren, of which some are known since long. In the following we list some of those of its special cases where the function ${ }_{3} F_{2}$ can be expressed in a more closed form, and some other consequences.

5. 1. Bateman's expansion. Putting in (38)

$$
\begin{array}{ll}
\rho=k r^{2}, & \tau=\cos 2 \vartheta, \\
\alpha=\cos ^{2} \varphi / 4 k, & \beta=\sin ^{2} \varphi / 4 k
\end{array}
$$


and letting $k \rightarrow 0$, we obtain, using (12) and replacing $2 \mu$ and $2 \nu$ by $\mu$ and $\nu$ respectively,

$(r \cos \varphi \cos \vartheta)^{-\mu} J_{\mu}(r \cos \varphi \cos \vartheta) \cdot(r \sin \varphi \sin \vartheta)^{-\nu} J_{\nu}(r \sin \varphi \sin \vartheta)$

$$
\begin{aligned}
=\sum_{m=0}^{\infty}(-)^{m} 2 m !(\mu+\nu+1+2 m) \Gamma(\mu+\nu+1+m) & \Gamma(\mu+1+m) \Gamma(\nu+1+m) \\
& \quad \times P_{m}^{(\nu, \mu)}(\cos 2 \varphi) P_{m}^{(\nu, \mu)}(\cos 2 \vartheta) r^{-\mu-\nu-1} J_{\mu+\nu+1+m}(r),
\end{aligned}
$$

which is equivalent to Bateman's expansion for the product of two Bessel functions [13, p. 370]. As pointed out by Watson, a great number of theorems on Bessel functions can be considered as special cases of this expansion.

5. 2. Product of Bessel functions, second case. If $\alpha=\beta=0$, we have, using a theorem by Watson [1, p. 16],

$$
{ }_{3} F_{2}\left[\begin{array}{cc}
-m,-m-2 \mu, \nu+\frac{1}{2} \\
-\mu-m+\frac{1}{2}, 2 \nu+1
\end{array}\right]= \begin{cases}\left(\begin{array}{l}
1 \\
2
\end{array}\right)_{n}(\mu+\nu+1+n)_{n} \\
(\nu+1)_{n}\left(\mu+\frac{1}{2}+n\right)_{n}, & \text { if } m=2 n \\
0, & \text { if } m=2 n+1, \\
n=0,1,2, \cdots,\end{cases}
$$

and thus by (11), after dividing by a numerical factor and replacing $\rho$ by $2 \rho$,

$$
\begin{aligned}
&\left(\begin{array}{c}
1+\tau \\
2
\end{array}\right)^{-\mu} I_{\mu}\left(\rho \frac{1+\tau}{2}\right) \cdot\left(\rho \begin{array}{c}
1-\tau \\
2
\end{array}\right)^{-\nu} I_{\nu}\left(\begin{array}{c}
1-\tau \\
2
\end{array}\right) \\
&=V 2 \Gamma\left(\mu+\nu+\frac{1}{2}\right) \\
&=\Gamma(\mu+1) \Gamma(\nu+1) \sum_{n=0}^{\infty}\left(\begin{array}{l}
1 \\
2
\end{array}\right)_{n}\left(\mu+\nu+\frac{1}{2}+2 n\right)\left(\mu+\nu+\frac{1}{2}\right)_{n} \\
&(\mu+1)_{n}(\nu+1)_{n} \\
& \times P_{2 n}{ }^{(2 \nu, 2 \mu)}(\tau) \rho^{-\mu-\nu-\frac{1}{2}} I_{\mu+\nu+\frac{1}{2}+2 n}(\rho) .
\end{aligned}
$$

This Neumann series for the product of two Bessel functions cannot be deduced from Bateman's expansion. The special case $\mu=\nu$ of it has been given by us already earlier [9, p. 333].

5. 3. Product of two Bessel functions, third case. Replacing in (38) $\rho, \alpha, \beta$ by $k \rho, 1 / 4 k,-1 / 4 k$, respectively and letting $k \rightarrow 0$, we obtain in view of (14), writing again $\mu, \nu$ instead of $2 \mu, 2 \nu$, 


$$
\begin{aligned}
\left(\rho \frac{1+\tau}{2}\right)^{-\frac{\mu}{2}} J_{\mu} & \left(\sqrt{\rho} \begin{array}{c}
1+\tau \\
2
\end{array}\right) \cdot\left(\begin{array}{c}
1-\tau \\
2
\end{array}\right)^{-\frac{\nu}{2}} I_{\nu}\left(\sqrt{\rho} \frac{1-\tau}{2}\right) \\
= & 2^{-\mu-\nu} \sum_{n=0}^{\infty} \Gamma(\mu+n+1) \Gamma(\nu+n+1)\left(\begin{array}{l}
\rho \\
4
\end{array}\right)^{n} P_{n}^{(\nu, \mu)}(\tau)
\end{aligned}
$$

Equivalent forms of this formula are well known in the theory of Bessel functions. ${ }^{7}$

5. 4. J.-W. expansion of a single Whittaker function. In the case $\beta=\nu+\frac{1}{2}$ the ${ }_{3} F_{2}$ in (38) reduces to 1 (one of its numerator parameters being zero) and the second of the two $M$-functions on the left becomes an exponential function. Thus we have

$$
\begin{aligned}
& e^{-\rho} \frac{1-\tau}{4}\left(\begin{array}{c}
1+\tau \\
2
\end{array}\right)^{-\mu-\frac{1}{2}} M_{\alpha, \mu}\left(\begin{array}{c}
1+\tau \\
2
\end{array}\right) \\
& =\sum_{m=0}^{\infty} \frac{\left(\mu+\frac{1}{2}-\alpha\right)_{m}}{(2 \mu+1)_{m}(2 \mu+2 \nu+1+m)_{m}} P_{m}^{(2 \mu, \nu)}(\tau) \rho^{-\mu-\nu-1} M_{\mu+\nu+\frac{1}{2}, \mu+\nu+\frac{1}{2}+m}(\rho) .
\end{aligned}
$$

An expansion which is equivalent to this one is listed by Buchholz [6, p. 130], who gives credit for it to Erdélyi. Buchholz also indicates various special cases of the expansion. ${ }^{8}$

5. 5. Product of two Whittaker functions, Remanujan's case. Another case in which the function ${ }_{3} F_{2}$ can be summed elementarily is given by the conditions $\alpha=\beta, \mu=\nu$. Then we have, upon application of a theorem by Dixon [1, p. 13]

$$
{ }_{3} F_{2}\left[\begin{array}{l}
-2 \mu-m, \mu+\frac{1}{2}-\alpha,-m ; \\
-\mu+\alpha-m+\frac{1}{2}, 2 \mu+1
\end{array}\right]=\left\{\begin{array}{r}
\frac{(2 n) !\left(\mu+\frac{1}{2}-\alpha\right)_{n}\left(\mu+\frac{1}{2}+\alpha\right)_{n},}{n !\left(\mu+\frac{1}{2}-\alpha\right)_{2 n}(2 \mu+1)_{n}} \\
\quad \text { if } m=2 n, \\
n=0,1,2, \cdots,
\end{array}\right.
$$

furthermore $[11$, p. 80$]$

$$
P_{n}^{(2 \mu, 2 \mu)}(\tau)=\frac{(2 \mu+1)_{n}}{(4 \mu+1)_{n}} C_{n}^{2 \mu+\frac{1}{2}}(\tau),
$$

${ }^{7}$ See, for example, [13, p. 148]; or for the special case $\mu=\nu=0$ also [12, p. 2].

${ }^{8}$ The case where the $M$-functions in the summation reduce to Bessel functions has (with $\tau=1$ ) been rediscovered recently by Slater $[\mathbf{1 6}]$. 
where $C_{n}^{2 \mu+\frac{1}{2}}$ denotes the Gegenbauer polynomial. Thus (38) becomes ${ }^{9}$

$$
\begin{aligned}
& \left(\rho^{2} \frac{1-\tau^{2}}{4}\right)^{-\mu-\frac{1}{2}} M_{\alpha, \mu}\left(\rho \frac{1+\tau}{2}\right) M_{\alpha, \mu}\left(\rho \frac{1-\tau}{2}\right) \\
& =\sum_{n=0}^{\infty} \frac{(2 n) !\left(\mu+\frac{1}{2}-\alpha\right)_{n}\left(\mu+\frac{1}{2}+\alpha\right)_{n}}{n !(2 \mu+1)_{n}(4 \mu+1)_{4 n}} C_{2 n}^{2 \mu+\frac{1}{2}}(\tau) \rho^{-2 \mu-1} M_{2 \alpha, 2 \mu+\frac{1}{2}+2 n}(\rho) .
\end{aligned}
$$

For $\tau=0$ we obtain in view of

$$
C_{2 n}^{2 \mu+\frac{1}{2}}(0)=(-)^{n} \frac{\left(2 \mu+\frac{1}{2}\right)_{n}}{n !}
$$

after multiplying by $(\rho / 2)^{2 \mu+1}$ and replacing $\rho$ by $2 \rho$ the series

$$
\left[M_{\alpha, \mu}(\rho)\right]^{2}
$$

$$
=2^{-2 \mu-1} \sum_{m=0}^{\infty} \frac{(-)^{n}(2 n) !\left(\mu+\frac{1}{2}-\alpha\right)_{n}\left(\mu+\frac{1}{2}+\alpha\right)_{n}\left(2 \mu+\frac{1}{2}\right)_{n}}{(n !)^{2}(2 \mu+1)_{n}(4 \mu+1)_{4 n}} M_{2 \alpha^{\prime} 2 \mu+\frac{1}{2}+2 n}(2 \rho)
$$

which expresses the square of an $M$-function as a series of $M$-functions in which the first index and the argument are duplicated. Expressing $\rho$ and $\tau$ on both sides of (43) by $z$ and $z^{*}$ and putting $z^{*}=0$, we have in view of Lemma 2, using (37) on the left.

$$
\begin{gathered}
{ }_{1} F_{1}\left[\begin{array}{l}
\mu+\frac{1}{2}-\alpha ; \frac{z^{2}}{4} \\
2 \mu+1
\end{array}\right]{ }_{1} F_{1}\left[\begin{array}{l}
\mu+\frac{1}{2}-\alpha ;-\frac{z^{2}}{4} \\
2 \mu+1
\end{array}\right] \\
=\sum_{n=0}^{\infty} \frac{\left(\mu+\frac{1}{2}-\alpha\right)_{n}\left(\mu+\frac{1}{2}+\alpha\right)_{n}}{(2 \mu+1)_{2 n}(2 \mu+1)_{n} n !}\left(\frac{z^{2}}{4}\right)^{2 n} \\
={ }_{2} F_{3}\left[\begin{array}{l}
\mu+\frac{1}{2}-\alpha, \mu+\frac{1}{2}+\alpha ; \frac{z^{4}}{64} \\
\mu+\frac{1}{2}, \mu+1,2 \mu+1
\end{array}\right] .
\end{gathered}
$$

This result was already found by Ramanujan [1, p. 97].

5. 6. Generalisation of Erdély's integral. Assuming $\Re \mu>-\frac{1}{2}$, $\Re_{\nu}>-\frac{1}{2}$, multiplying (38) by

${ }^{9}$ The special case $\mu=\alpha=\frac{1}{4}$ of this formula has been given in a different notation by Rainville [15]. (The $M$-functions on the left can then be expressed in terms of the error function.) Some misprints in [15] are pointed out in [14]. 


$$
(\rho / 2)^{\mu+\nu+1}(1-\tau)^{2 \nu}(1+\tau)^{2 \mu} P_{n}^{(2 \nu, 2 \mu)}(\tau),
$$

where $n$ is a fixed nonnegative integer and integrating with respect to $\tau$ from -1 to +1 we obtain in view of the well-known orthogonality properties of the Jacobi polynomials [11, p. 67]

$$
\begin{gathered}
\int_{-1}^{1}(1+\tau)^{\mu-\frac{1}{2}} M_{\alpha, \mu}\left[\begin{array}{c}
1+\tau \\
2
\end{array}\right] \cdot(1-\tau)^{\nu-\frac{1}{2}} M_{\beta, \nu}\left[\begin{array}{c}
1-\tau \\
2
\end{array}\right] P_{n}^{(2 \nu, 2 \mu)}(\tau) d \tau \\
=\frac{2^{\mu+\nu} \Gamma(2 \nu+n+1) \Gamma(2 \mu+1)\left(\mu+\frac{1}{2}-\alpha\right)_{n}}{n ! \Gamma(2 \mu+2 \nu+2 n+2)} \\
\quad \times{ }_{3} F_{2}\left[\begin{array}{l}
-2 \mu-n, \nu+\frac{1}{2}-\beta,-n ; \\
-\mu+\alpha-n+\frac{1}{2}, 2 \nu+1
\end{array}\right] \cdot M_{\alpha+\beta, \mu+\nu+\frac{1}{2}+n}(\rho) .
\end{gathered}
$$

For $n=0$ this reduces to a formula equivalent to a well-known result due to Erdélyi [8, p. 134]; see also [6, p. 128]. It is then most easily proved by means of the Laplace transformation.

5. 7. Neumann series for the product of two $M$-functions. We mention finally that the special case obtained by putting $\beta=-\alpha, \mu=\nu$ has been given by us already earlier (See [9, p. 329], and [10, p. 270], where also some special cases are discussed). In this case (and also in the more general case $\mu \neq \nu$ ) the $M$-functions on the right of (38) reduce to Bessel functions, without this being the case for the $M$ functions on the left.

\section{Applications of the Expansion Theorem: JaCOBIAN Elliptic CoORdinates}

Other particular solutions of (1) can be found by introducing in (4) or (16) new variables $\xi$ and $\eta$ defined by

$$
\xi=\widetilde{\omega}+\rho, \quad \eta=\widetilde{\omega}-\rho
$$

where

$$
\widetilde{\omega}=\sqrt{\left(\alpha-z^{2}\right)\left(\alpha-z^{* 2}\right)}=\sqrt{\alpha^{2}-2 \alpha \rho \tau+\rho^{2}},
$$

with some real constant $\alpha$, the square roots being positive for $z=z^{*}=0 .^{10}$

${ }_{10}$ These coordinates can be shown to be a special case of the general ellipsoidal coor. dinates, as investigated by Jacobi. Their use is also suggested by the structure of the generating function of the Jacobi polynomials. 
By elementary computations one finds that if $U\left(z, z^{*}\right)=W(\zeta, \eta),(16)$ is transformed into

$$
\begin{aligned}
& \left(\xi^{2}-\alpha^{2}\right) \frac{\partial^{2} W}{\partial \xi^{2}}-\left(\gamma^{2}-\alpha^{2}\right) \frac{\partial^{2} W}{\partial \eta^{2}}+2[(1+\mu+\nu) \xi-(\mu-\nu) \alpha] \frac{\partial W}{\partial \xi} \\
& -2[(1+\mu+\nu) \eta+(\mu-\nu) \alpha] \frac{\partial W}{\partial \eta}+k\left[(\xi+\eta) \frac{\lambda}{2}-\left(\xi^{2}-\eta^{2}\right) \frac{k}{16}\right] W=0 .
\end{aligned}
$$

This equation can again be separated. One finds by the usual method that if $W(\xi, \eta)=\Xi(\xi) H(\eta)$ is a solution, $\Xi$ and $H$ have to satisfy separately the two ordinary differential equations

$$
\begin{aligned}
& \left(\xi^{2}-\alpha^{2}\right) \frac{d^{2} \Xi}{d \xi^{2}}+2[(1+\mu+\nu) \xi-(\mu-\nu) \alpha] \frac{d \Xi}{d \xi}+k\left[p+\frac{\lambda}{2} \xi-\frac{k}{16} \xi^{2}\right] \Xi=0, \\
& \left(\eta^{2}-\alpha^{2}\right) \frac{d^{2} H}{d \eta^{2}}+2[(1+\mu+\nu) \eta+(\mu-\nu) \alpha] \frac{d H}{d \eta}+k\left[p-\frac{\lambda}{2} \eta-\frac{k}{16} \eta^{2}\right] H=0,
\end{aligned}
$$

where $p$ is a separation parameter. In order to obtain solutions of these equations in terms of known functions, it seems necessary to simplify them by assigning special values to some of the parameters. Two such simplifications will be indicated below, one of them leading again to Whittaker functions, the other to ordinary hypergeometric functions.

6. 1. Addition theorem for Whittaker functions. If in (50) we set $p=0, \nu=-\frac{1}{2}, \lambda=\frac{\alpha k}{8}$, the first equation becomes divisible by $\xi-\alpha$ and the second by $\eta+\alpha$. Cancelling these factors and setting (without essential loss of generality) $k=1$, we obtain the two equations

$$
\left.\begin{array}{l}
(\xi+\alpha) \frac{d^{2} \Xi}{d \xi^{2}}+(2 \mu+1) \frac{d \Xi}{d \xi}-\frac{\xi}{16} \Xi=0, \\
(\eta-\alpha) \frac{d^{2} H}{d \eta^{2}}+(2 \mu+1) \frac{d H}{d \eta}-\frac{\eta}{16} H=0,
\end{array}\right)
$$

which again can be easily reduced to Whittaker's equation. Carrying out the reduction one finds that solutions which as functions of $z$ and $z^{*}$ are regular near $z=z^{*}=0$, that is regular near $\xi=\eta=\alpha$, are given by

$$
\left.\begin{array}{l}
\Xi(\xi)=\left(\frac{\alpha+\xi}{2}\right)^{-\mu-\frac{1}{2}} M_{\frac{\alpha}{8}, \mu}\left(\frac{\alpha+\xi}{2}\right), \\
H(\eta)=\left(\frac{\alpha-\eta}{2}\right)^{-\mu-\frac{1}{2}} M_{\frac{\alpha}{8}, \mu}\left(\frac{\alpha-\eta}{2}\right),
\end{array}\right\}
$$


and that, as long as $2 \mu$ is not a negative integer, the product of these two functions belongs to $E \mathcal{K}_{\alpha} \times \mathcal{K}_{\alpha}$. Since for $z^{*}=0$

$$
\xi=\eta=\sqrt{\alpha^{2}-\alpha z^{2}},
$$

the generating function of the J.-W. expansion of $W(\xi, \eta)=\Xi(\xi) H(\eta)$ is

$$
f(z)=\left(\frac{\alpha z^{2}}{4}\right)^{-\mu-\frac{1}{2}} M_{\frac{\alpha}{8}, \mu}\left(\begin{array}{c}
\alpha+\sqrt{\alpha^{2}-\alpha z^{2}} \\
2
\end{array}\right) M_{-\frac{\alpha}{8}, \mu}\left(\begin{array}{c}
\alpha-\sqrt{\alpha^{2}-\alpha z^{2}} \\
2
\end{array}\right) .
$$

If we write

$$
f(z)=\sum_{m=0}^{\infty} A_{m}{ }^{(\mu)}(\alpha) z^{2 m},
$$

the required J.-W. expansion, valid in $\mathscr{K}_{\alpha} \times \mathscr{K}_{\alpha}$ and provided $2 \mu$ is not a negative integer, is

$$
\begin{aligned}
\left(\alpha \rho \frac{1+\tau}{2}\right)^{-\mu-\frac{1}{2}} & M_{\frac{\alpha}{8}, \mu}\left(\frac{\alpha+\rho+\widetilde{\omega}}{2}\right) M_{\frac{\alpha}{8}, \mu}\left(\frac{\alpha+\rho-\widetilde{\omega}}{2}\right) \\
& =\sum_{m=0}^{\infty} A_{m}{ }^{(\mu)}(\alpha) \frac{2^{2 m} m !}{(2 \mu+m)_{m}} P_{m}^{(-1,2 \mu)}(\tau) \rho^{-\mu-\frac{1}{2}} M_{-\frac{\alpha}{8}, \mu+m}(\rho),
\end{aligned}
$$

where $\widetilde{\omega}$ is given by (48). It does not seem possible to express the coefficients $A_{m}{ }^{(\mu)}$ in any closed form. Using a result of the previous section it is however not difficult to derive for them a series whose general term is again a $M$-function and whose coefficients can be exhibited explicitly. If in (43) we replace $\rho, \tau, \alpha$ by $\alpha, \sqrt{1-z^{2}} / \alpha, \alpha / 8$ respectively we obtain on the left just (53) and have therefore

$$
=\sum_{n=0}^{\infty} \frac{(2 n) !\left(\mu+\frac{1}{2}-\frac{\alpha}{8}\right)_{n}\left(\mu+\frac{1}{2}+\frac{\alpha}{8}\right)_{n}}{n !(2 \mu+1)_{n}(4 \mu+1)_{t n}} C_{2 n}^{2 \mu+\frac{1}{2}\left(\sqrt{\left.1-z^{2} / \alpha\right)}\right.} \alpha^{-2 \mu-1} M_{\frac{\alpha}{4}, 2 \mu+\frac{1}{2}+2 m}(\alpha) .
$$

Now, by Gauss' quadratic transformation,

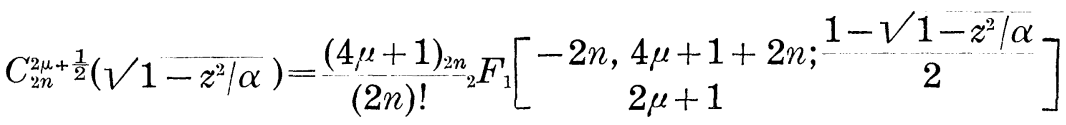

$$
\begin{aligned}
& =\frac{(4 \mu+1)_{2 n} F_{1}}{(2 n) !}\left[\begin{array}{c}
-n, 2 \mu+\frac{1}{2}+n ; z^{2} / \alpha \\
2 \mu+1
\end{array}\right]
\end{aligned}
$$

Inserting this in (56) and rearranging the series by collecting equal powers of $z$ (which is permissible in view of Weierstrass' theorem) we 
obtain in view of (54) the desired series representation

$$
\begin{gathered}
A_{m}{ }^{(\mu)}(\alpha)=\frac{(-\alpha)^{-m}\left(2 \mu+\frac{1}{2}\right)_{n} 2^{2 n}\left(\mu+\frac{1}{2}-\frac{\alpha}{8}\right)_{n}\left(\mu+\frac{1}{2}+\frac{\alpha}{8}\right)_{n}\left(2 \mu+\frac{1}{2}+m\right)_{n}}{(2 \mu+1)_{m} m ! \sum_{n=m}(n-m) !(4 \mu+1)_{4 n}} \\
\times \alpha^{-2 \mu-1} M_{\frac{\alpha}{4}, 2 \mu+\frac{1}{2}+2 n}(\alpha) .
\end{gathered}
$$

Since in virtue of this formula (56) expresses the product of the two functions

$$
M_{\frac{\alpha}{8}, \mu}\left(\frac{\alpha+\rho+\varepsilon \widetilde{\omega}}{2}\right), \quad \varepsilon= \pm 1
$$

in terms of products of $M$-functions with the arguments $\rho$ and $\alpha$ respectively, (55) may be looked at as an addition theorem for the functions on the left in analogy to a similar situation in the case of the well-known addition theorems of Graf and Gegenbauer in the theory of Bessel functions [13, p. 358].

For $\tau=-1$ we obtain from (55) the following addition theorem of a more elementary character:

$$
(\alpha+\rho)^{-\mu-\frac{1}{2}} M_{\frac{\alpha}{8}, \mu}(\alpha+\rho)=\sum_{m=0}^{\infty} \frac{(-)^{m} 2^{2 m}(2 \mu+1)_{m}}{(2 \mu+m)_{m}} A_{m}{ }^{(\mu)}(\alpha) \rho^{-\mu-\frac{1}{2}} M_{\frac{\alpha}{8}, \mu+m}(\rho)
$$

6. 2. Hypergeometric functions. Inserting in (51) the special values $p=q / k, \quad \alpha=1$, and letting $k \rightarrow 0$ yields the two differential equations

$$
\left\{\begin{array}{l}
\left(\xi^{2}-1\right) \frac{d^{2} \Xi}{d \xi^{2}}+2[(1+\mu+\nu) \xi-(\mu-\nu)] \frac{d \Xi}{d \xi}-q \Xi=0, \\
\left(\eta^{2}-1\right) \frac{d^{2} H}{d \eta^{2}}+2[(1+\mu+\nu) \eta+(\mu-\nu)] \frac{d H}{d \eta}-q H=0,
\end{array}\right.
$$

which are of hypergeometric type. Using this fact it is readily proved by substitution that solutions of these equations regular near $z=z^{*}=0$ (that is regular near $\xi=\eta=1$ ) are given by

(60)

$$
\left\{\begin{array}{c}
\Xi(\xi)={ }_{2} F_{1}\left[\begin{array}{c}
\left.\mu+\nu+\frac{1}{2}-\gamma, \mu+\nu+\frac{1}{2}+\gamma ; \frac{1-\xi}{2}\right] \\
2 \nu+1
\end{array}\right], \\
H(\eta)={ }_{2} F_{1}\left[\begin{array}{c}
\mu+\nu+\frac{1}{2}-\gamma, \mu+\nu+\frac{1}{2}+\gamma, \frac{1-\eta}{2} \\
2 \mu+1
\end{array}\right],
\end{array}\right.
$$


where

$$
\gamma=\sqrt{ }(2 \mu+2 \nu+1)^{2}-q
$$

is arbitrary, since $q$ was arbitrary. Writing $U\left(z, z^{*}\right)=\Xi(\xi) H(\eta)$ anb

$$
U(z, 0)=\sum_{n=0}^{\infty} a_{n} z^{2 n},
$$

we have, according to the expansion theorem,

$$
U\left(z, z^{*}\right)=\sum_{n=0}^{\infty} a_{n} F_{n}^{(\mu, \nu)}\left(z, z^{*} ; 0,0\right) .
$$

Since here the parameters $\lambda$ and $k$ are both zero, the coefficients $a_{n}$ can be easily determined by putting $z=z^{*}$. From (47) one has in this case $\xi=1, \eta=1-2 z^{2}$ and from (14), since now $\tau=1, \rho=z^{2}$,

$$
F_{n}{ }^{(\mu, \nu)}(z, z ; 0,0)=\frac{(2 \mu+2 \nu+1)_{n}(2 \nu+1)_{n}}{\left(\mu+\nu+\frac{1}{2}\right)_{n}(\mu+\nu+1)_{n}} z^{2 n} .
$$

Thus (61) reduces to

$$
{ }_{2} F_{1}\left[\begin{array}{c}
\mu+\nu+\frac{1}{2}-\gamma, \mu+\nu+\frac{1}{2}+\gamma ; z^{2} \\
2 \mu+1
\end{array}\right]=\sum_{n=0}^{\infty} a_{n} \frac{(2 \mu+2 \nu+1)_{n}(2 \nu+1)_{n} z^{2 n},}{\left(\mu+\nu+\frac{1}{2}\right)_{n}(\mu+\nu+1)_{n}},
$$

which yields

$$
a_{n}=\frac{\left(\mu+\nu+\frac{1}{2}-\gamma\right)_{n}\left(\mu+\nu+\frac{1}{2}+\gamma\right)_{n}\left(\mu+\nu+\frac{1}{2}\right)_{n}(\mu+\nu+1)_{n}}{(2 \mu+1)_{n}(2 \nu+1)_{n}(2 \mu+2 \mu+1)_{n} n !} .
$$

Thus (62) may now be stated more explicitly as follows:

$$
\begin{gathered}
{ }_{2} F_{1}\left[\mu+\nu+\frac{1}{2}-\gamma, \mu+\nu+\frac{1}{2}+\gamma ; \frac{1-\rho+\tilde{\omega}}{2}\right] \\
2 \nu+1 \\
\times{ }_{2} F_{1}\left[\mu+\nu+\frac{1}{2}-\gamma, \mu+\nu+\frac{1}{2}+\gamma ; \frac{1+\rho-\widetilde{\omega}}{2}\right] \\
2 \mu+1 \\
=\sum_{n=0}^{\infty} \frac{\left(\mu+\nu+\frac{1}{2}-\gamma\right)_{n}\left(\mu+\nu+\frac{1}{2}+\gamma\right)_{n} \rho^{n} P_{n}^{(2 \nu, 2 \mu)}(\tau) .}{(2 \mu+1)_{n}(2 \nu+1)_{n}}
\end{gathered}
$$


A result equivalent to this was derived by Brafman ${ }^{11}$ from Bailey's decomposition formula for a special case of Appell's hypergeometric function $F_{4}$ of two variables [1, p. 81.] Since it is also possible to derive Bailey's formula from (65) simply by replacing the Jacobi polynomial by its hypergeometric definition and inserting appropriate values of $\rho$ and $\tau$, our proof of (65) contains also a new proof of that formula.

Restating (61) with the explicit value of $a_{n}$ given by (64) we obtain

$$
{ }_{2} F_{1}\left[\begin{array}{c}
\left.\mu+\nu+\frac{1}{2}-\gamma, \mu+\nu+\frac{1}{2}+\gamma ; \frac{1-\sqrt{1-z^{2}}}{2}\right] \\
2 \mu+1
\end{array}\right.
$$

$$
\begin{aligned}
& \times_{2} F_{1}\left[\begin{array}{c}
\left.\mu+\nu+\frac{1}{2}-\gamma, \mu+\nu+\frac{1}{2}+\gamma ; \frac{1-V 1-z^{2}}{2}\right] \\
2 \nu+1
\end{array}\right] \\
& ={ }_{4} F_{3}\left[\begin{array}{c}
\mu+\nu+\frac{1}{2}-\gamma, \mu+\nu+\frac{1}{2}+\gamma, \mu+\nu+\frac{1}{2}, \mu+\nu+1 ; z^{2} \\
2 \mu+1,2 \nu+1,2 \mu+2 \nu+1
\end{array}\right],
\end{aligned}
$$

which is equivalent to a result proved by Bailey $[1$, p. 88 , formula (3)] by means of transformations of terminating generalized hypergeometric series.

\section{REFERENCES}

1. W. N. Bailey, Generalized hypergeometric series, Cambridge, 1935.

2. S. Bergman, The kernel function and conformal mapping, Amer. Math Soc., 1950.

3. On solutions with algebraic character of linear partial differential equations, Trans. Amer. Math. Soc. 68 (1950), 461-507.

4. On solutions of linear partial differential equations of mixed type, J. Amer. Math. Soc. 74 (1952), 444-474.

5. F. Brafman, Generating functions of Jacobi and related polyncmials. Proc. Amer. Math. Soc. 2 (1951), 942-949.

6. H. Buchholz, Die konfluente hypergecmetrische Funktion, Berlin, Göttingen, Heidelberg, 1953.

7. R. Courant, and D. Hilbert, Methoden der mathematischen Physik, Bd. 2., Berlin, 1937.

8. A. Erdélyi, Funktionalrelationen mit konfluenten hypergeometrischen Funktionen, Erste Mitteilung, Math. Z. 42 (1937), 125-143.

9. P. Henrici, A Neumann series for the product of tuo Whittaker functions, Proc. Amer. Math Soc. 4 (1953), 329-334.

${ }^{11}$ See [5, p. 943], where also numerous special cases of the formula involving Gegenbauer and Legendre polynomials are given. 
10. Zur Funktionentheorie der Wellengleichung, Comm. Math. Helv. 27 (1953), 235-293.

11. G. Szegö, Orthogonal polynomials, New York, 1939.

12. E. Truesdell, An essay toward an unified theory of special functions, Princeton, 1918.

13. G. N. Watson, Treatise on the theory of Bessel functions, 2nd. ed. Cambridge and New York, 1944.

14. J.C. Cooke, Note on a heat conduction problem. Amer. Math. Monthly 62 (1955), 331-334.

15. E. D. Rainville, A heat conduction problem and the product of two error functions. J. Math. Physics 32 (1953), 43-47.

16. L. J. Slater, Expansions of generalized Whittaker functions. Proc. Cambr. Phil. Soc. 50 (1954), 628-631.

National Bureau of Standards, Washington, D.C. 


\section{PACIFIC JOURNAL OF MATHEMATICS}

\section{EDITORS}

\author{
H. L. Royden \\ Stanford University \\ Stanford, California \\ E. Hewite \\ University of Washington \\ Seattle 5 , Washington
}

\author{
R. P. Dilworth
}

California Institute of Technology Pasadena 4, California

\section{A. HorN*}

University of California

Los Angeles 24, California

\section{ASSOCIATE EDITORS}

\author{
H. BUSEMANN \\ HERBERT FEDERER \\ MARSHALL HALL
}

\author{
P. R. HALMOS \\ HEINZ HOPF \\ ALFRED HORN
}

\author{
R. D. JAMES \\ BORGE JESSEN \\ PAUL LÉVY
}

GEORGE PÓLYA

J. J. STOKER

KOSAKU YOSIDA

\section{SPONSORS}

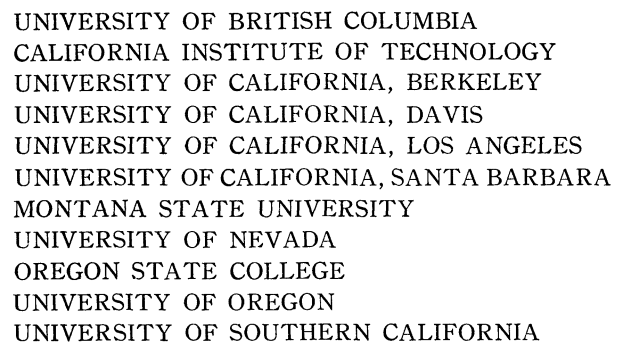

UNIVERSITY OF BRITISH COLUMBIA

CALIFORNIA INSTITUTE OF TECHNOLOGY

UNIVERSITY OF CALIFORNIA, BERKELEY

UNIVERSITY OF CALIFORNIA, DAVIS

UNIVERSITY OF CALIFORNIA, LOS ANGELES

UNIVERSITY OF CALIFORNIA, SANTA BARBARA

MONTANA STATE UNIVERSITY

UNIVERSITY OF NEVADA

OREGON STATE COLLEGE

UNIVERSITY OF OREGON

UNIVERSITY OF SOUTHERN CALIFORNIA

\author{
STANFORD RESEARCH INSTITUTE \\ STANFORD UNIVERSITY \\ UNIVERSITY OF UTAH \\ WASHINGTON STATE COLLEGE \\ UNIVERSITY OF WASHINGTON
}

AMERICAN MATHEMATICAL SOCIETY

HUGHES AIRCRAFT COMPANY SHELL DEVELOPMENT COMPANY

Mathematical papers intended for publication in the Pacific Journal of Mathematics should be typewritten (double spaced), and the author should keep a complete copy. Manuscripts may be sent to any of the editors. Manuscripts intended for the outgoing editors should be sent to their successors. All other communications to the editors should be addressed to the managing editor, Alfred Horn at the University of California Los Angeles 24, California.

50 reprints of each article are furnished free of charge; additional copies may be obtained at cost in multiples of 50 .

The Pacific Journal of Mathematics is published quarterly, in March, June, September, and December. The price per volume (4 numbers) is $\$ 12.00$; single issues, $\$ 3.50$; back numbers (Volumes $1,2,3)$ are available at $\$ 2.50$ per copy. Special price to individual faculty members of supporting institutions and to individual members of the American Mathematical Society: $\$ 4.00$ per volume; single issues, $\$ 1.25$.

Subscriptions, orders for back numbers, and changes of address should be sent to the publishers, University of California Press, Berkeley 4, California.

Printed at Kokusai Bunken Insatsusha (International Academic Printing Co., Ltd.) No. 10 1-chome Fujimi-cho, Chiyoda-ku, Tokyo, Japan.

* During the absence of E. G. Straus. 


\section{Pacific Journal of Mathematics}

\section{Vol. 5, No. $5 \quad$ BadMonth, 1955}

Henry A. Antosiewicz, A theorem on alternatives for pairs of matrice . . . . . 641

F. V. Atkinson, On second-order non-linear oscillation ............... 643

Frank Herbert Brownell, III, Fourier analysis and differentiation over real separable Hilbert spac .................................. 649

Richard Eliot Chamberlin, Remark on the averages of real function ...... 663

Philip J. Davis, On a problem in the theory of mechanical quadrature ... . . 669

Douglas Derry, On closed differentiable curves of order $n$ in $n$-spac ...... 675

Edwin E. Floyd, Boolean algebras with pathological order topologie ... . . 687

George E. Forsythe, Asymptotic lower bounds for the fundamental frequency of convex membrane ................................. 691

Israel Halperin, On the Darboux propert ................... 703

Theodore Edward Harris, On chains of infinite orde .............. 707

Peter K. Henrici, On certain series expansions involving Whittaker functions

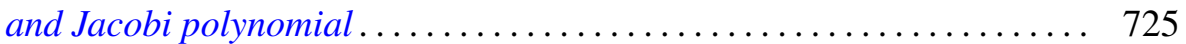

John G. Herriot, The solution of Cauchy's problem for a third-order linear hyperoblic differential equation by means of Riesz integral ......... 745

Jack Indritz, Applications of the Rayleigh Ritz method to variational

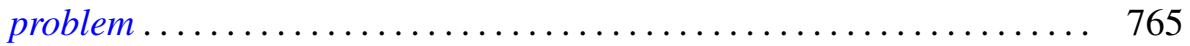

E. E. Jones, The flexure of a non-uniform bea ................. 799

Hukukane Nikaidô and Kazuo Isoda, Note on non-cooperative convex game.

Raymond Moos Redheffer and W. Wasow, On the convergence of asymptotic solutions of linear differential equation . . .

S. E. Warschawski, On a theorem of L. Lichtenstei ...........

Philip Wolfe, The strict determinateness of certain infinite game... 